05,12

\title{
Проявление низкоразмерного магнетизма в $K \alpha$-спектрах C, N, O карбонатов и нитратов, полученных при рентгеноспектральном микрозондовом анализе (РСMA)
}

\author{
(С) И.М. Куликова, О.А. Набелкин, В.А. Иванов, И.А. Филенко \\ Институт минералогии, геохимии и кристаллохимии редких элементов, \\ Москва, Россия \\ E-mail: imkulikova@gmail.com, mulderfw@yandex.ru
}

Поступила в Редакцию 25 декабря 2020 г.

В окончательной редакции 17 мая 2021 г.

Принята к публикации 18 мая 2021 г.

\begin{abstract}
Зарегистрированы первичные $\mathrm{C} K \alpha$-, $\mathrm{N} K \alpha$ - и ОK $\alpha$-спектры монокристаллов карбонатов, нитритов и нитратов с помощью метода рентгеноспектрального микрозондового анализа (РСМА). Сдвиг и ширину $K \alpha$-линий использовали для установления закономерностей изменений зарядовой и спиновой плотности на излучающем атоме в низкоразмерных магнетиках. Метод позволил экспериментально получить представления о наличии косвенного обменного взаимодействия в исследованных образцах монокристаллах (в том числе микронных размеров) при комнатной температуре. В спектрах азота в нитратах и нитрите в полосе проводимости выявлена структура резонансных уровней (плазмонов).
\end{abstract}

Ключевые слова: $K \alpha$-спектры углерода, азота, кислорода, карбонаты, нитраты, нитриты, спиновая плотность, зарядовая плотность, суперобмен, рентгеноспектральный микрозондовый анализ.

DOI: 10.21883/FTT.2021.10.51397.274

\section{1. Введение}

Низкоразмерные магнетики являются объектами усиленного экспериментального и теоретического изучения [1-4]. В таких системах спины магнитных ионов взаимодействуют преимущественно вдоль одного или двух направлений по отношению к кристаллографическим осям и существенно слабее в других. Данная работа посвящена изучению явлений с проявлением низкоразмерного магнетизма в карбонатах переходных металлов и нитрате натрия. Нитраты и, особенно, карбонаты проявляют большую склонность к образованию соединений с протяженной структурой. Магнитные ионы в этих соединениях взаимодействуют между собой через карбонатные $\mathrm{CO}_{3}^{2-}$ или нитратные $\mathrm{NO}_{3}^{-}$анионы (косвенное обменное взаимодействие).

Мы сообщаем результаты о магнитных свойствах исследованных образцов монокристаллов (минералов и синтетических соединений), полученные с помощью рентгеноспектрального микрозондового анализа (РСМА) при исследовании закономерности изменения зарядовой и спиновой электронной плотности атомов $\mathrm{C}$, $\mathrm{N}, \mathrm{O}$, излучающих первичные $K \alpha$-спектры. $K \alpha$-спектры атомов $\mathrm{C}, \mathrm{N}, \mathrm{O}$ возникают при переходе исследуемого атома из начального состояния с вакансией в $1 s$-уровне в конечное состояние с вакансией в $2 p$-уровне валентной полосы. Наличие рентгеновской вакансии - это сильное локальное возмущение. Поэтому $K \alpha$-линия несет информацию об электронной структуре излучающего атома, который в „Z + 1-приближении“ эквивалентен примеси, находящейся в центре кластера, и о влиянии на ее атомов ближайших одной или двух координационных сфер.
При комнатных температурах на близких расстояниях в пределах кластеров магнитный порядок сохраняется за счет обменных сил, хотя в кристалле общий магнитный порядок разрушается. Зарядовая и спиновая плотность такого атома зависит как от величины „мгновенной“ (средней по состояниям системы) ориентации спинов атомов ближайшего окружения, так и от плотности состояний в зоне проводимости. В окрестности подобной „Примеси“ может образоваться резонансный уровень, заполняемый при экранировании потенциала внутренней вакансии электронами зоны проводимости. Время жизни состояний с внутренней вакансией в $K \alpha$-спектрах ( $1 \mathrm{fs})$ мало по сравнению с теоретическим пределом для времени спиновой переориентации (2 ps [5]). В этом заключено преимущество рентгеновской спектроскопии перед резонансными методами, с помощью которых из-за большого периода прецессии ядерного спина $(\sim 10 \mathrm{~ns})$ удается исследовать только среднее по времени значение спина электронов. Существенным преимуществом анализа с помощью метода РСМА является локальность излучающей поверхности, позволяющая изучать монокристаллы микронных размеров.

Ранее в некоторых ромбоэдрических антиферромагнетиках $\left(\alpha-\mathrm{Fe}_{2} \mathrm{O}_{3}, \mathrm{MnCO}_{3}, \mathrm{CoCO}_{3}\right.$ и др.) была обнаружена аномальная зависимость магнитной восприимчивости от магнитного поля [6-8]; теоретическое объяснение аномалии было дано Дзялошинским [9]. А именно, при установлении вдоль тригональной оси антиферромагнитного упорядочения у ряда магнитных структур, в которых все магнитные ионы в элементарной ячейке занимают кристаллографически эквивалентные позиции, возникает слабый ферромагнитный момент, направленный 
перпендикулярно к тригональной оси [6,7]. Известно, что слабый ферромагнетизм антиферромагнетиков, при котором устанавливается неколлинеарное упорядочение спинов, является весьма распространенным явлением.

Структура исследованных карбонатов - минералов группы кальцита (кальцит $\mathrm{CaCO}_{3}$, родохрозит $\mathrm{MnCO}_{3}$, сферокобальтит $\mathrm{CoCO}_{3}$ ) относится к ромбоэдрической сингонии $C \overline{3} m$. Каждая группа $\mathrm{CO}_{3}$ состоит из трех атомов кислорода, образующих равносторонний треугольник, в центре которого расположен атом углерода. Расстояния между атомами кислорода в треугольнике составляют около $2.3 \AA$. Все группы $\mathrm{CO}_{3}$ лежат в плоскостях, перпендикулярных тройной оси $c$ [10]. В минералах группы кальцита атомы металла находятся в вершинах ячейки и в ее центре, две группы $\mathrm{CO}_{3}$, связанные одна с другой центром инверсии, расположены на центральной тройной оси.

Кутнагорит $\mathrm{CaMn}\left(\mathrm{CO}_{3}\right)_{2}$ относится к группе доломита, которую представляют двойные углекислые соли Са, $\mathrm{Mg}$, Fe и $\mathrm{Mn}$. Кристаллизуются эти минералы в ромбоэдрическом виде симметрии тригональной сингонии $C \overline{3}$. Структура кутнагорита аналогична структуре кальцита, но вдоль каждой из тройных осей атомы Са через один заменены атомами $\mathrm{Mn}$. В доломите $\mathrm{CaMg}\left(\mathrm{CO}_{3}\right)_{2}$ наличие двух различных катионов приводит к превращению плоского карбонат-иона в пирамидальный с высотой $0.018 \AA$ и развороту его на $6.35^{\circ}$ относительно оси третьего порядка [11].

Нитрат натрия $\mathrm{NaNO}_{3}$ кристаллизуется в виде симметрии тригональной сингонии. В $\mathrm{NO}_{3}$-группе три атома О располагаются в вершинах треугольника с короткими расстояниями О-O $(\approx 2.17 \AA)$ и с ионом $\mathrm{N}^{5+}$ в центре, лежащем с ними в одной плоскости. Кристаллические состояния нитрата аммония $\mathrm{NH}_{4} \mathrm{NO}_{3}$ $\beta$-ромбическая (бипирамидальная) в диапазоне температур от -16.8 до $32.3^{\circ} \mathrm{C}$ и $\alpha$-ромбическая (моноклинная) в диапазоне от 32.3 до $84.2^{\circ} \mathrm{C}$.

Определение химического состава исследуемых монокристаллов провели методом РСМА. В минералах группы кальцита содержания изоморфных примесей $\mathrm{Ca}$, $\mathrm{Mn}, \mathrm{Fe}, \mathrm{Co}, \mathrm{Ni}, \mathrm{Zn}$ не превышали $0.04 \%$ wt и только в сферокобальтите содержание $\mathrm{Ca}$, Fe и $\mathrm{Ni}$ составило, соответственно, $0.08,0.10$ и $0.15 \%$ wt. В кутнагорите установили наличие примеси $\mathrm{Fe}$ и $\mathrm{Ni}$ в количестве 0.18 и $0.24 \% \mathrm{wt}$, соответственно. В исследованных синтетических монокристаллах нитратов и нитрите примесных элементов не обнаружено.

В ряде ромбоэдрических антиферромагнетиков вдоль тригональной оси $c$ кристалла наблюдалась [3,6] нормальная зависимость намагниченности от величины магнитного поля. В то же время, В плоскости, перпендикулярной этой оси, при температурах ниже $T_{c}=31.5 \mathrm{~K}$ для $\mathrm{MnCO}_{3}$ и $T_{c}=17.5 \mathrm{~K}$ для $\mathrm{CoCO}_{3}$ магнитная восприимчивость резко возрастает и сильно зависит от напряженности поля. Острый максимум восприимчивости $\chi_{\perp}$ наблюдали в $\mathrm{CoCO}_{3}$. Величина спонтанного ферромагнитного момента составляла небольшую долю (от 0.1 до $10 \%$ ) номинального момента. Наблюдаемая аномалия объясняется тем, что ниже $T_{c}$ магнитные моменты двух магнитных ионов ориентируются не строго антипараллельно, а повернуты относительно друг друга на небольшой угол $\varphi$ (например, $\varphi=5^{\circ}$ для $\alpha-\mathrm{Fe}_{2} \mathrm{O}_{3}[8]$; наибольшим значением угла $\varphi \approx 7^{\circ}$ обладает $\left.\mathrm{CoCO}_{3}[3,6,7]\right)$. В ряду кристаллографически изоморфных карбонатов с ионами $\mathrm{Mn}^{2+}, \mathrm{Co}^{2+}, \mathrm{Ni}^{2+}$ и $\mathrm{Fe}^{2+}$ первые три обладают аномальными свойствами, в то время как $\mathrm{FeCO}_{3}$ ведет себя как обычный антиферромагнетик.

Цепочка косвенных обменных взаимодействий в магнитных диэлектриках с участием валентных оболочек немагнитных атомов (суперобмен) [7] может включать и более одного промежуточного иона. В металлах и сплавах косвенное магнитное взаимодействие может осуществляться через коллективизированные электроны проводимости (например, по $K \alpha_{1}$-линии титана в сплавах с ниобием, ванадием и молибденом установлен вклад в спиновую плотность на атоме Ті электронов полосы проводимости [12]). Это косвенное обменное взаимодействие может иметь дальнодействующий характер $[7,13]$. Во многих твердых телах магнитные ионы расположены достаточно далеко друг от друга, из-за чего непосредственное обменное взаимодействие между электронами магнитных атомов слабое.

\section{2. Рентгеноспектральный метод}

Известно, что сдвиг центра тяжести $K \alpha$-линии $\left(\delta E_{\text {c.g. }}\right)$ отражает изменение величины зарядовой плотности. Уширение $K \alpha$-линии $\left(\Gamma_{1 / 2}\right)$ диамагнитных ионов в значительной степени связано с проявлением мультиплетной структуры $2 p$-уровня, возникающей за счет косвенного обменного взаимодействия (сверхобмен) с парамагнитными ионами. Например, титанаты со структурой ильменита $M \mathrm{TiO}_{3}(M=\mathrm{Mn}, \mathrm{Fe}, \mathrm{Co}, \mathrm{Ni})$ обладают антиферромагнитными свойствами, которые индуцируют появление слабого ферромагнетизма [14]. Структура ильменита (гексагональная пространственная группа $R \overline{3}$ ) с катионами $\mathrm{Fe}^{2+}$ и $\mathrm{Ti}^{4+}$, полностью упорядоченными вдоль оси $c$, близка к структуре гематита $\alpha-\mathrm{Fe}_{2} \mathrm{O}_{3}$. Ранее, в работе [15] установили, что параметры внутренней $K \alpha_{1}$-линии диамагнитного иона $\mathrm{Ti}^{4+}$ (вторичные спектры) в титанатах со структурой ильменита (рис. 1) $\mathrm{FeTiO}_{3}$ (ильменит) и $\mathrm{MnTiO}_{3}$ (пирофанит) занимают промежуточное положение между параметрами спектров диамагнитных гейкилита $\mathrm{MgTiO}_{3}$ (рутила $\mathrm{TiO}_{2}$ ) и парамагнитного $\mathrm{Ti}_{2} \mathrm{O}_{3}$ (эффективный магнитный момент $\left.\mu_{\mathrm{eff}}\left(\mathrm{Ti}^{3+}\right)=1.8 \mu_{\mathrm{B}}[13]\right)$. Это свидетельствует о наличии в $\mathrm{FeTiO}_{3}$ и $\mathrm{MnTiO}_{3}$ спиновой плотности на титане за счет косвенного обменного взаимодействия через $2 p$-орбитали кислорода с магнитными ионами $\mathrm{Fe}^{2+}, \mathrm{Mn}^{2+}$, находящимися во второй координационной сфере. Долю неспаренной спиновой плотности на титане в $\mathrm{FeTiO}_{3}$ и $\mathrm{MnTiO}_{3}$ оценили как 0.33 и 0.24, соответственно [15]. 


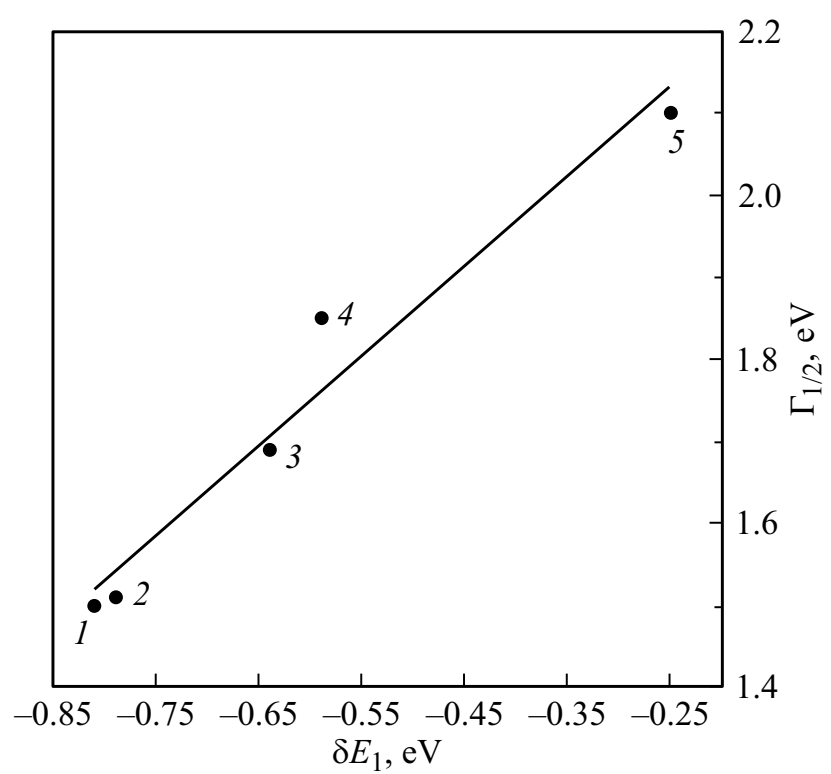

Рис. 1. Изменения ширины $\Gamma_{1 / 2}$ и сдвига $\delta E_{1} K \alpha_{1}$-линии Тi (относительно чистого металла Ті) в соединениях $\mathrm{MgTiO}_{3}(1)$, $\mathrm{TiO}_{2}$ (2), $\mathrm{MnTiO}_{3}$ (3), $\mathrm{FeTiO}_{3}$ (4) и $\mathrm{Ti}_{2} \mathrm{O}_{3}$ (5) [15]. Точность определения параметров $\pm 0.02 \mathrm{eV}[12]$.

В работе [16] рентгеновские эмиссионные ОK $\alpha$-спектры были использованы для получения информации о характере электронных взаимодействий атомов кислорода нитроксильной и нитронной групп: о величине зарядовой и спиновой плотности валентной полосы.

\section{3. Экспериментальная часть}

Приведенные ниже аналитические и технические параметры анализа получены на микрозондовом анализаторе „Camebax-microbeam“ (Франция) с кристалломанализатором ODPB $(2 d=99.98 \AA)$, при ускоряющем напряжении $10 \mathrm{kV}$ в режиме растра [17]. Чтобы исключить образование на поверхности образцов электрического заряда, максимальный ток пучка электронов при измерении в режиме растра $20 \times 20 \mu \mathrm{m}^{2}$ ограничивали величиной $50 \mathrm{nA}$, а при растре $40 \times 40 \mu \mathrm{m}^{2}$ величиной 120-150 nA. При использовании в качестве образцов сравнения $\mathrm{N}, \mathrm{O}$ и $\mathrm{C}$ синтетических $\mathrm{BN}_{\text {cub }}, \mathrm{Fe}_{2} \mathrm{O}_{3}$ и ультрадисперсных алмазов статического синтеза (с линейным размером 20-40 $\mu \mathrm{m})$, они, а также и образцы минералов, были упакованы в шашку из эпоксидной смолы и отполированы. Монокристаллы нитрита и нитрата натрия, нитрата аммония с линейными размерами 3-5 mm, проявляющие в поле свободных электронов зонда сегнетоэлектрическую неустойчивость [18], крепили на двусторонний углеродный скотч; на поверхность кристаллов наносили тонкий токопроводящий слой антистатика (аэрозоль). Чтобы сбалансировать спонтанную поляризацию монокристаллов, ток пучка электронов выбирали конкретно для каждого образца в
Таблица 1. Энергии связи $E_{1 s}($ в $\mathrm{eV})$ в исследованных соединениях [19]

\begin{tabular}{c|c|c|c}
\hline Соединение & $\mathrm{N} 1 s$ & $\mathrm{O} 1 s$ & $\mathrm{C} 1 s$ \\
\hline$\left(\mathrm{NH}_{2}\right)_{2} \mathrm{CO}$ & 400.0 & 531.9 & 289.4 \\
\hline $\mathrm{NaNO}_{2}$ & 404.0 & 532.9 & - \\
\hline $\mathrm{NaNO}_{3}$ & 407.3 & 533.0 & - \\
\hline$\left(\mathrm{NH}_{4}\right) \mathrm{NO}_{3}$ & $402.0^{*}, 407.4^{* *}$ & 533.1 & - \\
\hline $\mathrm{BN}$ & 398.2 & - & - \\
\hline $\mathrm{Fe}_{2} \mathrm{O}_{3}$ & - & 530.2 & - \\
\hline алмаз & - & - & 285.0 \\
\hline СаСО & - & - & 289.3
\end{tabular}

При и ечан и е. ${ }^{*}$ Атом азота в ионе $\mathrm{NH}_{4}^{+} .{ }^{* *}$ Атом азота в ионе $\mathrm{NO}_{3}^{-}$.

области линейного изменения интенсивности аналитических линий [17]. Для измерений выбрали размер растра $40 \times 40 \mu \mathrm{m}^{2}$ с перемещением образца под пучком электронов в пределах площадки $\approx 120 \times 120 \mu \mathrm{m}^{2}$ под контролем отсутствия кратеров повреждения поверхности образцов в оптическом микроскопе (увеличение $\times 500)$. Все спектры - результат усреднения $3-4^{\mathrm{x}}$ экспериментальных спектрограмм, полученных в спектральном диапазоне, разбитом на равные участки (50-85 точек).

Спектр кислорода изучался во 2-ом порядке отражения от кристалла-анализатора. Энергия максимумов $K \alpha$-линий $\mathrm{C}, \mathrm{O}, \mathrm{N}$ используемых образцов сравнения ультрадисперсных алмазов статического синтеза, гематита $\mathrm{Fe}_{2} \mathrm{O}_{3}$ и $\mathrm{BN}_{\text {cub }}$, - равна 279.5, 525.0 и $396.7 \mathrm{eV}$, соответственно. Величины энергии связи внутренних $1 s$-уровней атомов $\mathrm{N}, \mathrm{O}, \mathrm{C}$, использованные для представления спектров различных элементов в единой энергетической шкале, в которой ноль соответствует уровню вакуума, приведены в табл. 1 .

\section{4. Результаты и их обсуждение}

В исследуемых соединениях конечные состояния $\mathrm{C} K \alpha$-, $\mathrm{N} K \alpha$-, ОK $\alpha$-спектров связанны с вакансией в $2 p$-уровне валентной полосы и поэтому спектры имеют большую ширину по сравнению с шириной внутренних $K \alpha_{1}$-линий $3 d$-элементов (например, в титанатах, ванадатах $[12,15])$. Валентная зона карбонатов и нитратов имеет преимущественно анионный характер; внутри анионных групп $\mathrm{CO}_{3}^{2-}$ и $\mathrm{NO}_{3}^{-}$реализуется ковалентная связь, а между анионом и катионом связь в значительной степени ионная. Порядок следования уровней в анионах $\mathrm{CO}_{3}^{2-}$ и $\mathrm{NO}_{3}^{-}$следующий [20]:

$$
\begin{array}{ccc}
\text { „О2 } 2 s^{“-у р у р о в н и ~} & \sigma \text {-уровни } & \text {,О } 2 p_{\pi} \text { “-уровни } \\
1 a_{1}^{\prime} 1 e^{\prime} & 2 a_{1}^{\prime} 2 e^{\prime}\left(1 a_{2}^{\prime \prime} \pi \text {-связь }\right) & \left(4 e^{\prime} 1 e^{\prime \prime} 1 a_{2}^{\prime}\right)
\end{array}
$$




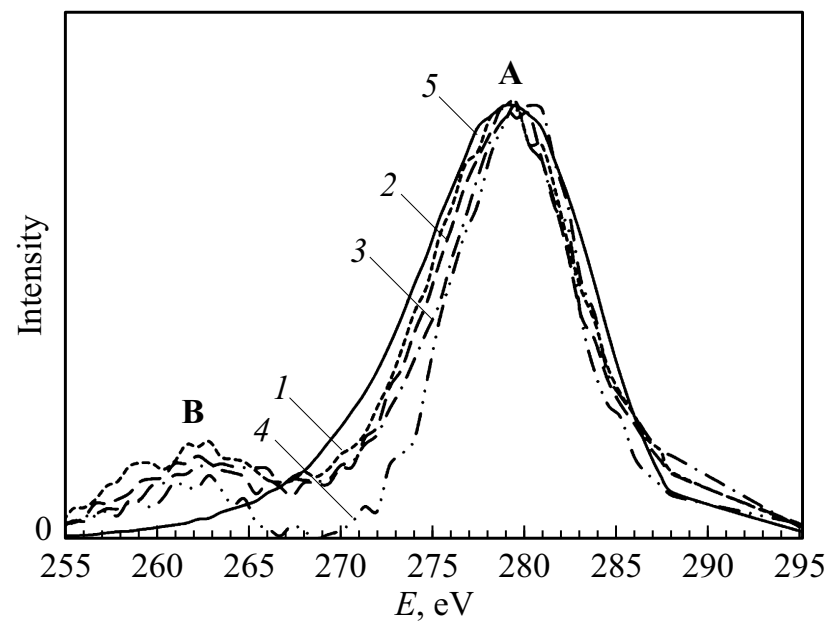

Рис. 2. $K \alpha$-спектры углерода в образцах: $\mathrm{CoCO}_{3}$ (1), $\mathrm{MnCO}_{3}$ (2), $\mathrm{CaCO}_{3}$ (3), $\mathrm{CaMn}\left(\mathrm{CO}_{3}\right)_{2}$ (4), алмаз (5). Спектры на рисунке нормированы по интенсивности.

В рамках теории функционала локальной электронной плотности вычислено [21,22], что в карбонатах (нитратах) валентная полоса образована гибридизованными $s$ - и $p$-состояниями атомов аниона $\mathrm{CO}_{3}^{2-}\left(\mathrm{NO}_{3}^{-}\right)$. Самый верх валентной области образован полностью $\mathrm{O} 2 p$-состояниями, в середине - зоны с наибольшей степенью гибридизации состояний $\mathrm{C}(\mathrm{N})$ и $\mathrm{O}$, ниже расположены зоны О2s-орбиталей. В изоэлектронном ряду $\mathrm{CO}_{3}^{2-} \rightarrow \mathrm{NO}_{3}^{-}$разности энергий валентных уровней увеличиваются, и, как следствие, значительно вырастает ширина $K \alpha$-линии азота по сравнению с $K \alpha$-линией углерода.

\section{1. Карбонаты}

На рис. 2 показаны С $K \alpha$-линии (за вычетом фона) в различных карбонатах (спектры 1-4), полученные в настоящем исследовании: максимум $\mathbf{A}-$ уров-
Таблица 2. Параметры $K \alpha$-линий азота и кислорода: $E_{\text {c.g. }}$ и $\Gamma_{1 / 2}($ в $\mathrm{eV})$

\begin{tabular}{|c|c|c|c|c|c|}
\hline \multirow{2}{*}{\multicolumn{2}{|c|}{ Соединение }} & \multicolumn{2}{|c|}{$\mathrm{O}$} & \multicolumn{2}{|c|}{$\mathrm{N}$} \\
\hline & & $E_{\text {c.g. }}$ & $\Gamma_{1 / 2}$ & $E_{\text {c.g. }}$ & $\Gamma_{1 / 2}$ \\
\hline \multicolumn{2}{|c|}{$\left(\mathrm{NH}_{2}\right)_{2} \mathrm{CO}$} & - & - & 395.0 & 14.8 \\
\hline \multirow{2}{*}{$\begin{array}{l}\text { Образцы } \\
\text { сравнения }\end{array}$} & $\mathrm{Fe}_{2} \mathrm{O}_{3}$ & 524.5 & 10.5 & - & - \\
\hline & $\mathrm{BN}_{\mathrm{cub}}$ & - & - & 395.1 & 14.8 \\
\hline \multicolumn{2}{|c|}{$\mathrm{NaNO}_{3}$} & 524.2 & 12.5 & 396.5 & 18.1 \\
\hline \multicolumn{2}{|c|}{$\mathrm{NH}_{4} \mathrm{NO}_{3}$} & 526.5 & 11.5 & 398.1 & 14.8 \\
\hline
\end{tabular}

ни $\pi+\sigma$, максимум $\mathbf{B}-$ примесь $\mathrm{C} 2 p$-состояний к О $2 s$-орбиталям.

В табл. 2 представлены параметры спектров $\mathrm{N} K \alpha$ и $\mathrm{O} K \alpha$ монокристаллов исследованных азотсодержащих соединений: энергетические положения центров тяжести $E_{\text {c.g. }}$ и величины ширины $K \alpha$-линий на половине высоты $\Gamma_{1 / 2}$.

Величину параметра $\eta$, характеризующую относительную интегральную интенсивность спектров 2 -электронов атомов $\mathrm{C}, \mathrm{N}, \mathrm{O}$, сопоставили с $\Gamma_{1 / 2}$ в исследуемых соединениях (рис. 3). В методе РСМА параметр $\eta$ определяли следующим образом: $\eta=\left(S_{x} / I_{x}\right) /\left(S_{\mathrm{ref}} / I_{\mathrm{ref}}\right)$, где $I_{\mathrm{ref}}, I_{x}$ - интенсивность излучения (за вычетом фона) в максимуме аналитической линии в образце сравнения и в исследуемом образце, соответственно; $S_{\text {ref }}, S_{x}-$ суммарная интенсивность $K \alpha$-линии (за вычетом суммарного фона) по всем точкам измерения (50-85 точек). Из рис. 3 следует, что изменения величины $\eta$ являются показателем соответствия расщепления уровней основной полосы и уровней всего спектра (коэффициенты корреляции для С, N и О равны, соответственно, 0.88, 0.98 и 0.74$)$.

На рис. 4 представлен график изменения параметров

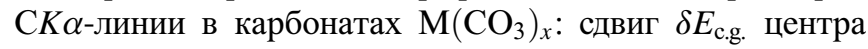
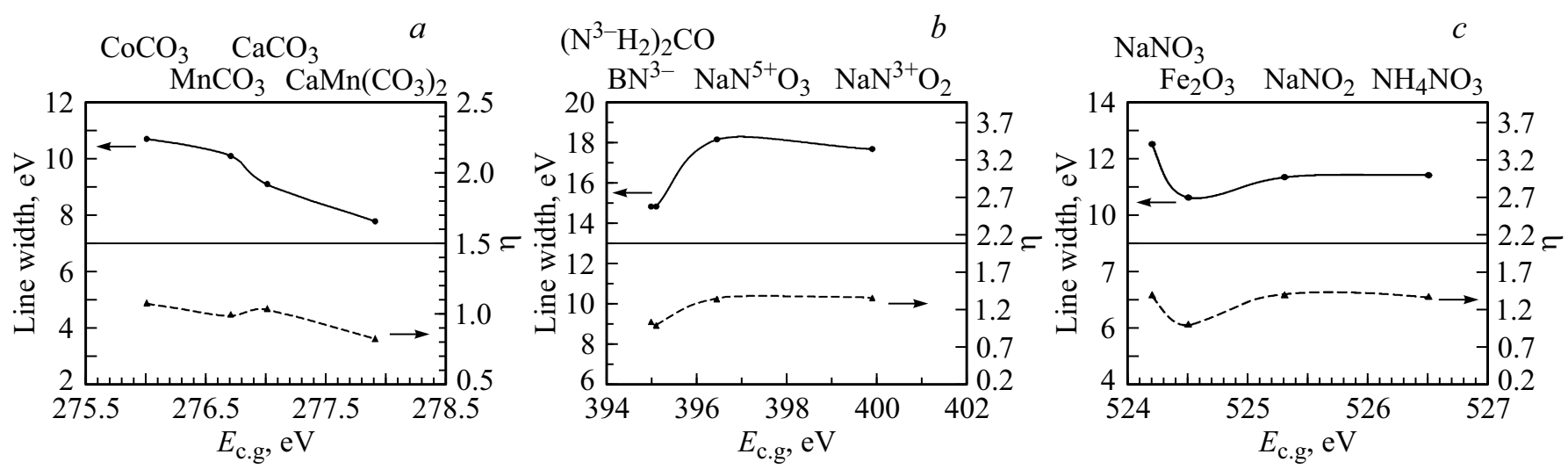

Рис. 3. Сопоставление изменений ширины $\Gamma_{1 / 2} \mathrm{C} K \alpha(a), \mathrm{N} K \alpha(b), \mathrm{O} K \alpha(c)$ и параметра $\eta$ при изменении центра тяжести $E_{\mathrm{c.g}}$ линий в указанных на рисунке соединениях. 
тяжести $K \alpha$-линий углерода относительно линии в алмазе и ширины $\Gamma_{1 / 2}$.

Характер изменения электронной структуры лигандов в этих соединениях позволил выделить следующие закономерности.

С ростом степени ионности связи углерода в кутнагорите $\mathrm{CaMn}\left(\mathrm{CO}_{3}\right)_{2}$ относительно алмаза резко уменьшается расщепление полосы А с 11.2 до $7.8 \mathrm{eV}$, появляется примесь С $2 p$-состояний к О $2 s$-состояниям (максимум В на рис. 2) при практически неизменной энергии центра тяжести $K \alpha$-линии (рис. 4). (Энергия связи $\mathrm{C} 1 s$ приведена в табл. 1).

В ряду карбонатов $M\left(\mathrm{CO}_{3}\right)_{x}$, где $M=$ $=(\mathrm{CaMn}) \rightarrow \mathrm{CaMn} \rightarrow \mathrm{Co}$ по изменениям парциальных вкладов в спектр углерода установлен рост электронного переноса между катионами $M^{2+}$ и анионами $\mathrm{CO}_{3}^{2-}$, а также внутри анионных групп между $\pi$ - и $\sigma$-полосами (в область низкоэнергетичного склона полосы А) (рис. 2). В этом ряду карбонатов увеличивается примесь $\mathrm{C} 2 p$ к $\mathrm{O} 2 s$ (максимум В). Результат дополнительного переноса $2 p$ электронной плотности представлен на рис. 5 выделением в области $\sigma$-полосы и в области $\mathrm{O} 2 s$ дополнительной разностной интенсивности (полосы $\alpha$ и $\beta$ ) в спектрах $\mathrm{CoCO}_{3}$ по сравнению с $\mathrm{CaMn}\left(\mathrm{CO}_{3}\right)_{2}$.

Известно [24], что в ряду катионов $M^{2+}$ повышение переноса электронной плотности от катионов к атомам кислорода в анионах $\mathrm{CO}_{3}^{2-}$ уменьшает их способность оттягивать электроны от рассматриваемого атома углерода. Таким образом и появилась в исследуемых карбонатах дополнительная, по сравнению с $\mathrm{CaMn}\left(\mathrm{CO}_{3}\right)_{2}$, электронная плотность углерода $\rho=\rho^{\alpha}+\rho^{\beta}$ (растет общая спаренная и неспаренная спиновая плотность). Вклад дополнительной интенсивности в спектр $2 p$-состояний углерода (плотности $\rho$ ) в $\mathrm{MnCO}_{3}$ и $\mathrm{CoCO}_{3}$ составляет 29.3 и 34.5\%, соответственно. Ростом $\rho$ обусловлен отрицательный сдвиг центра тяжести $K \alpha$-линии углерода в карбонатах (рис. 4) относительно $\mathrm{CaMn}\left(\mathrm{CO}_{3}\right)_{2}$.

В работе [21] из расчетов зонной структуры, плотности состояний карбонатов металлов установлено, что основным механизмом образования химической связи является электронный перенос между $\pi$ - и $\sigma$-орбиталями кислорода в связевую $\mathrm{C}-\mathrm{O}$ область и в области за драми атомов.

Рост ширин линий углерода $\Gamma_{1 / 2}$, а также разностных полос $\alpha, \beta$ (с 7.8 в $\mathrm{CaMn}\left(\mathrm{CO}_{3}\right)_{2}$ до 10.7 и $10.1 \mathrm{eV}$ в $\mathrm{CoCO}_{3}$ и $\mathrm{MnCO}_{3}$, соответственно) связан с проявлением мультиплетной структуры С2p-уровня, возникающей за счет сверхобмена атомов углерода с парамагнитными ионами $M^{2+}$ через $p$-орбитали кислорода (растет перекрытие $d$-орбиталей ионов $M^{2+}$ с $p$-орбиталями кислорода). Спиновая плотность $\rho_{s}$ на атомах углерода, возникающая при сверхобмене за счет $\sigma$-связей атомов углерода с $p$-орбиталями кислорода, $\propto \rho\left(\Gamma_{1 / 2} \propto \delta E_{\text {c.g. }}\right.$ углерода см. на рис. 4).

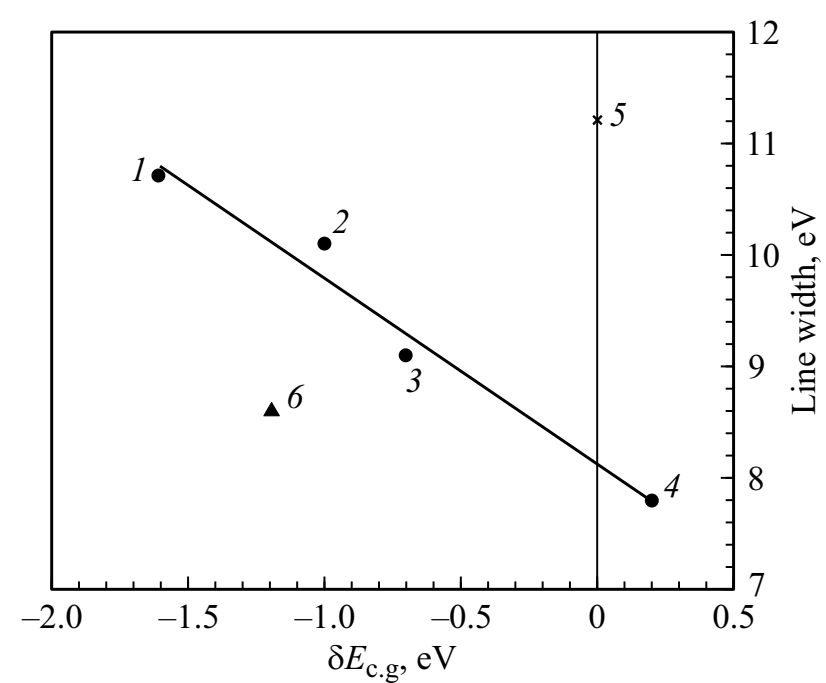

Рис. 4. Изменения параметров $\mathrm{C} K \alpha$-спектров в соединениях $\mathrm{CoCO}_{3}$ (1), $\mathrm{MnCO}_{3}$ (2), $\mathrm{CaCO}_{3}$ (3), $\mathrm{CaMn}\left(\mathrm{CO}_{3}\right)_{2}$ (4), алмазе (5) и карбамиде $\left(\mathrm{NH}_{2}\right)_{2} \mathrm{CO}(6)$. Стандартное отклонение величин $0.3 \mathrm{eV}$.

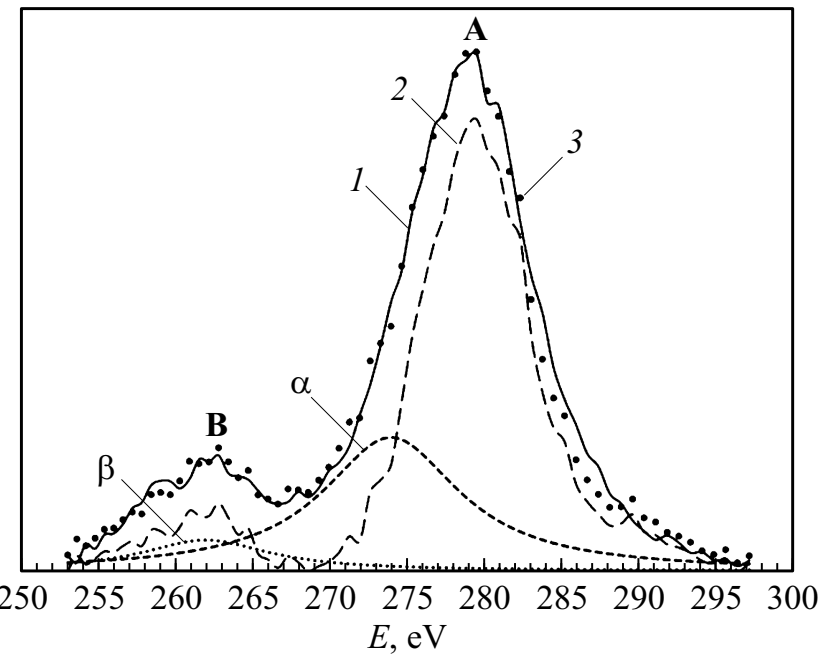

Pис. 5. $\mathrm{CK \alpha}$ спектры $\mathrm{CoCO}_{3}(1)$ и $\mathrm{CaMn}\left(\mathrm{CO}_{3}\right)_{2}$ (2). Суммарная интенсивность (3) линии $\mathrm{CK} \alpha$ в $\mathrm{CaMn}\left(\mathrm{CO}_{3}\right)_{2}$ и интенсивности полос $\alpha, \beta\left(\Gamma_{1 / 2}^{\alpha, \beta}=10.8 \mathrm{eV}\right.$, форма - спектральное распределение Лоренца).

В структуре исследованных карбонатов парамагнитные ионы $M^{2+}$ и анионы $\mathrm{CO}_{3}^{2-}$ расположены на одной прямой. В работе [1] было установлено, что магнитные взаимодействия (сила и тип ориентации магнитных моментов) и возможность их переориентации (переход антиферромагнетик $\leftrightarrow$ ферромагнетик) определяются в значительной степени геометрическим расположением и размером промежуточных ионов в локальном пространстве между двумя магнитными ионами. Так, при большем радиусе катиона $\mathrm{Co}^{2+}$ по сравнению с $\mathrm{Mn}^{2+}$ расщепление полосы $\mathbf{A}$ в спектре углерода в $\mathrm{CoCO}_{3}$ больше, чем в $\mathrm{MnCO}_{3}$ (рис. 4), несмотря на эффектив- 

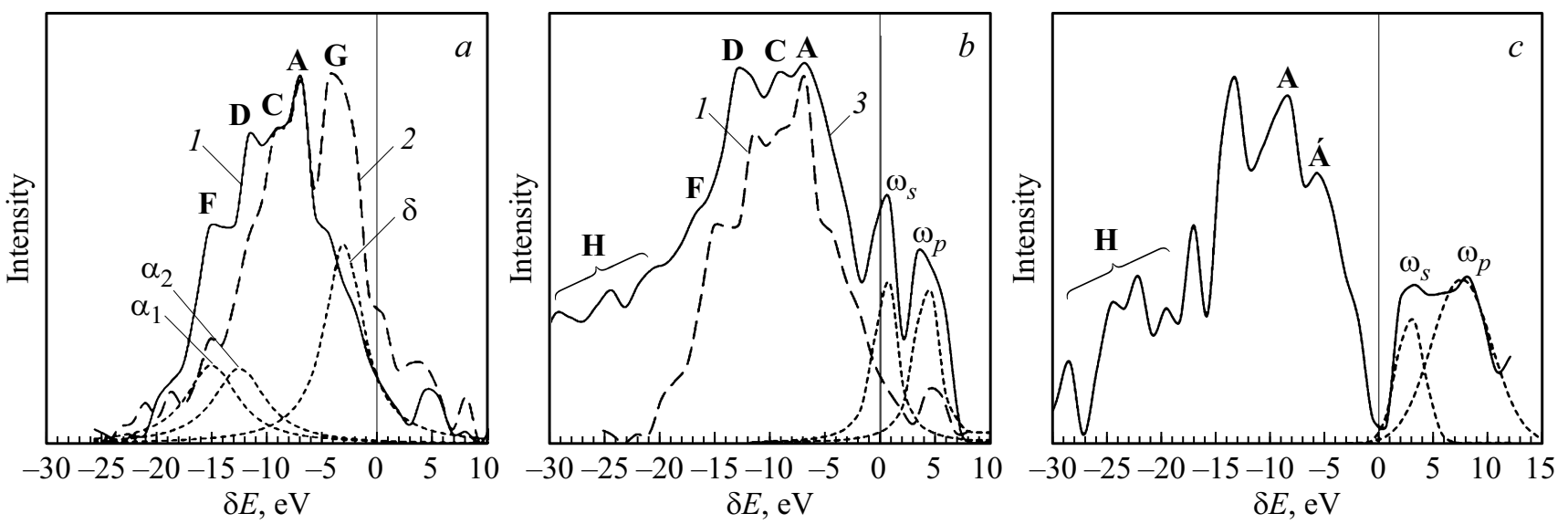

Pис. 6. ОK $\alpha$ спектры $\mathrm{NaNO}_{3}(1), \mathrm{NH}_{4} \mathrm{NO}_{3}(2), \alpha_{1}, \alpha_{2}$ и $\delta$ - разностные спектральные интенсивности в области максимумов F, D и $\mathbf{G}$ этих спектров $(a) ; \mathrm{N} K \alpha(3)$ и $\mathrm{O} K \alpha(1)$ в $\mathrm{NaNO}_{3}(b)$; линия $\mathrm{N} K \alpha$ в $\mathrm{NH}_{4} \mathrm{NO}_{3}(c)$; $\omega_{s}$ и $\omega_{p}$ - поверхностный и объемный плазмоны (распределение Лоренца $(b)$ и Гаусса $(c)$ ).

ные магнитные моменты $\mu_{\mathrm{eff}}$ ионов $\mathrm{Co}^{2+}$ и $\mathrm{Mn}^{2+}$, равные, соответственно, $4.8 \mu_{\mathrm{B}}$ и $5.9 \mu_{\mathrm{B}}[13]$.

В работе [24] на основе анализа экспериментальных данных показано, что сдвиги энергии внутреннего уровня $1 s$-электрона атома углерода в неорганических и органических соединениях могут быть представлены в виде аддитивной функции параметров, характеризующих связи атомов углерода с ближайшими атомами. Однако, как видно из рис. 4 , в кутнагорите $\mathrm{CaMn}\left(\mathrm{CO}_{3}\right)_{2}$ отсутствует аддитивность вкладов от Са и $\mathrm{Mn}$ не только в спиновой плотности на атомах углерода, но и в электронной плотности. Это - результат разрушения магнитного порядка в цепочке атомов, связанных обменным взаимодействием, при замене в структуре доломита, по сравнению с кальцитом, атомов Са через один атомами Mn вдоль каждой из тройных осей.

\section{2. Нитраты}

В работе [23] были исследованы некоторые особенности электронной структуры азотсодержащих соединений методом РСМА (параметры спектров $\mathrm{O}, \mathrm{N}$ представлены в табл. 2).

По своему положению последняя полоса $\mathbf{G}$ в $\mathrm{O} 2 p$ спектре нитрата аммония $\mathrm{NH}_{4} \mathrm{NO}_{3}$ (рис. 6, $a$ ), соответствующая электронной плотности, участвующей в $\pi$-связи, в $\mathrm{NaNO}_{3}$ резко уменьшается по интенсивности на величину $\delta$-полосы. При этом в области $\sigma$-полосы $\mathrm{NaNO}_{3}$ (максимумы D и $\mathbf{F}$ на рис. 6, $a$ ) появилась дополнительная электронная плотность кислорода $\rho_{\alpha_{1}}$ и $\rho_{\alpha_{2}}$ (расщепление $\boldsymbol{\alpha}_{1}$ и $\boldsymbol{\alpha}_{2}$ полос $2.5 \mathrm{eV}$, ширина каждой полосы в спектре $5.8 \mathrm{eV}$ ). Суммарная интегральная интенсивность полос $\boldsymbol{\alpha}_{1}$ и $\boldsymbol{\alpha}_{2}$ равна интегральной интенсивности $\delta$ полосы и составляет $26.6 \%$ от всего спектра $\mathrm{NaNO}_{3}$. Ширины $\boldsymbol{\alpha}_{1}$ и $\boldsymbol{\alpha}_{2}$ полос больше, чем у $\boldsymbol{\delta}$ полосы, ширина которой $4.2 \mathrm{eV}$. Как следствие, ширина $\Gamma_{1 / 2}$ линии $\mathrm{O} K \alpha$ в нитрате натрия увеличена на $2.0 \mathrm{eV}$ по сравнению с гематитом $\mathrm{Fe}_{2} \mathrm{O}_{3}$ и на $1.0 \mathrm{eV}$ по сравнению с нитратом аммония $\mathrm{NH}_{4} \mathrm{NO}_{3}$ и свидетельствует о спиновой поляризации электронной плотности на ионах кислорода в нитрате натрия. (В гематите $\alpha-\mathrm{Fe}_{2} \mathrm{O}_{3}$ в результате косвенного обменного взаимодействия между атомами железа $\left(\mu_{\mathrm{eff}}\left(\mathrm{Fe}^{3+}\right)=5.9 \mu_{\mathrm{B}}[13]\right.$ через кислород у атомов последнего имеется спиновая плотность.)

На рис. 6, $b$ совместили в единой энергетической шкале $K \alpha$-спектры азота и кислорода в нитрате натрия $\mathrm{NaNO}_{3}$. Соответствие положения парциальных вкладов $\mathbf{A}, \mathbf{C}, \mathbf{D}, \mathbf{F}$ азота и кислорода в спектрах определяет зону с наибольшей степенью гибридизации $2 p$-состояний $\mathrm{N}$ и О. В области затянутого низкоэнергетичного склона Н линии азота в нитратах $\mathrm{NaNO}_{3}$ и $\mathrm{NH}_{4} \mathrm{NO}_{3}$ (рис. 6,c) не удалось выделить примесь $\mathrm{N} 2 p$-состояний к О $2 s$-орбиталям (аналог максимуму В на рис. 2) изза наложения сателлитов, обусловленных неупругим рассеянием выбитого $1 s$-электрона [19].

Характерная особенность спектров атомов азота с формально положительной степенью окисления в монокристаллах $\mathrm{NaNO}_{3}, \mathrm{NH}_{4} \mathrm{NO}_{3}$ и $\mathrm{NaNO}_{2}$, исследованных в работе [23], - это наличие интенсивных дискретных максимумов $\omega_{s}$ и $\omega_{p}$ в тонком слое по границе полосы проводимости (рис. $6, b, c)$. Исследованные соединения характеризуются ковалентно-ионной связью; электропроводность чистого поликристаллического $\mathrm{NaNO}_{2}$ в сегнетоэлектрической фазе возрастает в 100 раз. ${ }^{1}$

Максимумы - спектры коллективных электронных возбуждений в полосе проводимости за счет экранирования потенциала $2 p$-вакансии атома азота в конечном состоянии при испускании им $K \alpha$-линии. Эти сателлиты в спектре азота соответствуют резонансам поверхностных и объемных плазмонов $\left(\omega_{s}\right.$ и $\omega_{p}$ на рис. $6, b$, соответственно). Параметры плазмонных резонансов в $\mathrm{NaNO}_{3}$

${ }^{1}$ Е.В. Стукова исследовала зависимость свойств сегнетоэлектрических материалов от спонтанной поляризации, наличия проводимости и размерных эффектов. Дис. докт. физ.-мат. наук (Диэлектрические свойства неоднородных микро- и наноразмерных сегнетоэлектрических систем. СПб, 2014). 

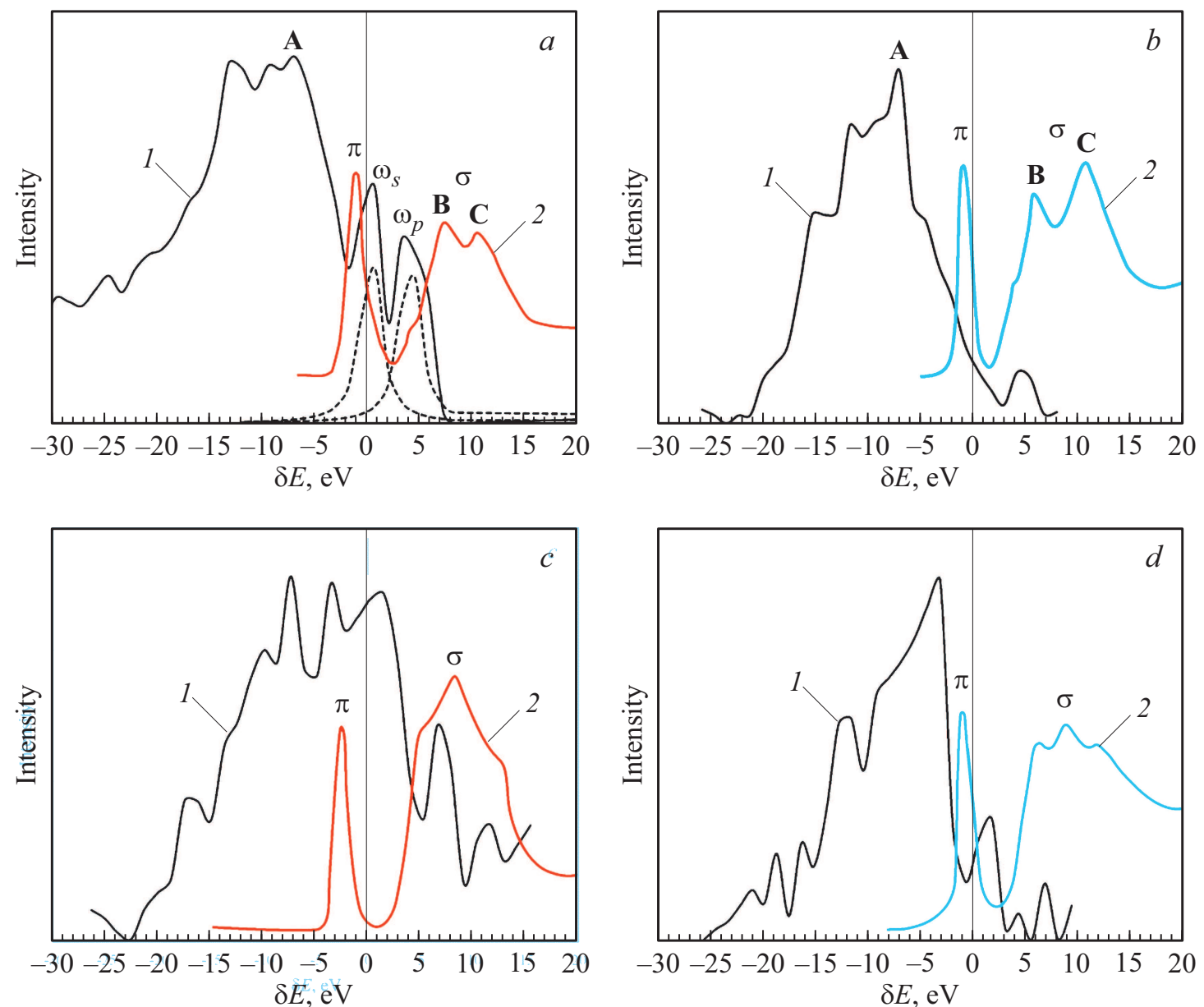

Pис. 7. Спектры азота $(a, c)$ и кислорода $(b, d)$ в $\mathrm{NaNO}_{3}$ поглощения (2) [26] в единой энергетической шкале.

и $\mathrm{NH}_{4} \mathrm{NO}_{3}$, определенные из спектров на рис. $6, b, c$, представлены в табл. 3. В этой таблице $\gamma-$ это ширина на половине высоты линии, $E_{\omega_{s}}$ и $E_{\omega_{p}}$ расстояния между высшим занятым уровнем валентной полосы $\mathbf{A}$ ( $\pi$-уровни аниона $\left.\mathrm{NO}_{3}^{-}\right)$и резонансными уровнями поверхностных и объемных плазмонов, соответственно, $\omega_{p} / \omega_{s}-$ отношение частот колебаний электронов объемных и поверхностных плазмонов. (Максимум Á на рис. 6, $c$ соответствует компоненте $\mathrm{NH}_{4}$-группы [23].) Величина $E_{\omega}$ поверхностного плазмона в $\mathrm{NaNO}_{3}$ соответствует экспериментальным данным начала роста фотоэмиссии $(7.5 \mathrm{eV})[25]$.

Из табл. 3 видно, что частота объемного плазмона $\omega_{p}$ в $\sim \sqrt{2}$ больше, чем частота $\omega_{s}$ поверхностного плазмона.

Аналогичные максимумы, локализованные в зоне проводимости $\mathrm{NaNO}_{3}, \mathrm{KNO}_{3}$ и $\mathrm{NaNO}_{2}$, близкие к резонансам формы и проявляющиеся в $K$-спектрах поглощения $\mathrm{N}$ и О, подробно исследовались В.Н. Сивковым. ${ }^{2}$

\footnotetext{
${ }^{2}$ В.Н. Сивков Дис. докт. физ.-мат. наук (Распределение сил осцилляторов в области резонансной структуры ультрамягких рентгеновских спектров поглощения молекул и твердых тел. СПб (2003).
}

На рис. 7 мы совместили по энергии $K \alpha$-спектры и $K$-края поглощения [26] азота $(7, a, c)$ и кислорода $(7, b, d)$ в $\mathrm{NaNO}_{3}$ и $\mathrm{NaNO}_{2}$. Из спектров азота прослеживается идентичность формы резонансов: в $\mathrm{NaNO}_{3}$ расстояние $E_{\omega_{p}}-E_{\omega_{s}}=3.5 \mathrm{eV}$ соответствует расстоянию между максимумами $\mathbf{B}-\mathbf{C}(\sigma$-резонансы $)$, проявляющимися в $K$-спектрах поглощения $\mathrm{N}$ [26]. В $\mathrm{NaNO}_{3}$ резонансы в спектрах поглощения жестко смещены относительно резонансов в эмиссионном спектре на $6.2 \mathrm{eV}$ (на $6 \mathrm{eV}$ в $\mathrm{NaNO}_{2}$ ); $\pi$-резонанс в спектре поглоще-

Таблица 3. Параметры плазмонных резонансов на рис. $6, b, c$ : $\gamma, E_{\omega_{s}}, E_{\omega_{p}}$ в $\mathrm{eV} ; \omega_{p} / \omega_{s}$

\begin{tabular}{l|c|c|c|c|c|c}
\hline \multirow{2}{*}{ Образец } & \multicolumn{3}{|c|}{$\mathrm{NaNO}_{3}$} & \multicolumn{3}{|c}{$\mathrm{NH}_{4} \mathrm{NO}_{3}$} \\
\cline { 1 - 4 } & $\gamma$ & $E_{\omega}$ & $\left(\omega_{p} / \omega_{s}\right)^{2}$ & $\gamma$ & $E_{\omega}$ & $\left(\omega_{p} / \omega_{s}\right)^{2}$ \\
\hline $\begin{array}{l}\text { Поверхностный } \\
\text { плазмон } \omega_{s}\end{array}$ & 2.3 & 7.3 & & 3.0 & 11.1 & \\
\cline { 1 - 2 } $\begin{array}{l}\text { Объемный } \\
\text { плазмон } \omega_{p}\end{array}$ & 2.3 & 10.8 & 2.19 & 6.5 & 15.8 & 2.03 \\
& & & & &
\end{tabular}


ния смещен на $5.2 \mathrm{eV}$ относительно максимума $\mathbf{A}$ в $K \alpha$-спектре. В $K \alpha$-спектрах атомов кислорода слабый резонанс выявлен только в полосе проводимости $\mathrm{NaNO}_{2}$ (см. рис. $7, d$ ), в отличие от резонансов формы в $K$-спектре поглощения О. Резонансы формы - метастабильное состояние системы, которое распадается путем туннелирования частицы, удерживаемой потенциальным барьером, сквозь него в континуум, или связано с „надбарьерным“ рассеянием фотоэлектрона. Контур резонансов существенно зависит от величины взаимодействия дискретного состояния с континуумом.

Плазменная частота $\omega$ определяется осцилляциями валентных электронов в металле по отношению к положительно заряженным остовам атомов и в большинстве металлов составляет порядка $1 \mathrm{fs}^{-1}$, что совпадает с обратной величиной времени жизни внутренней вакансии в $K \alpha$-спектрах. Из-за наличия релаксационных процессов направленное движение электронов плазмонов затухает. За эффекты релаксации, в первую очередь, ответственно взаимодействие между электронами проводимости и кристаллической структурой, которое существенно влияет на форму резонансной линии [13]. Затухание плазмонов определяется аддитивными механизмами потерь, т.е. полная ширина линии для каждого плазмона представляется в виде суммы трех компонент: объемными (выражаются через эффективную частоту соударений электронов), поверхностными (упругое отражение от границы плазмы) и радиационными потерями [27,28].

Если хундовская обменная энергия больше естественной ширины резонансного уровня „примесного“ атома, то на нем в полосе проводимости возникает спиновая плотность $\left(\rho_{s}^{\mathrm{imp}}\right)[13](\gamma$ в табл. 3 включает также аппаратурные искажения, достаточно большие при РСМА). Из измерений ширины $K \alpha$-линии мы получаем информацию о спиновой плотности $\rho_{s}$, представляющей собой сумму трех аддитивных вкладов: локального (обусловленного как потенциалом вакансии, так и собственным магнитным моментом, если он есть), дополнительного за счет обмена с соседними парамагнитными атомами и вклада, обусловленного спиновой поляризацией электронов в зоне проводимости.

Мы считаем, что уширение $K \alpha$-линии азота в $\mathrm{NaNO}_{3}$ $\left(\Gamma_{1 / 2}=18.1 \mathrm{eV}\right.$ в табл. 2) связано с мультиплетной структурой $2 p$-уровня, возникающей за счет спиновой плотности локального уровня, обусловленного потенциалом вакансии $\rho_{s}^{\text {imp }}$. Судя по ширине $\gamma$ в табл. 3, спиновая плотность $\rho_{s}^{\text {imp }}$ в нитрате натрия больше, чем в нитрате аммония и, как следует из табл. 2 (ширина $\Gamma_{1 / 2}$ ), больше спиновая поляризация $2 p$-электронов.

\section{5. Заключение}

При исследовании антиферромагнетиков при комнатной температуре с помощью рентгеновских спектров можно получить информацию о локальной спиновой плотности, так как время жизни состояний с внутренней вакансией в $K \alpha$-спектрах порядка 1 fs мало по сравнению со временем спиновой переориентации. Наиболее пригодны для исследования косвенного обменного взаимодействия формально диамагнитные ионы, так как в них отсутствует эффект от своих собственных неспаренных электронов. Основной вклад в спиновую плотность при косвенном обменном взаимодействии возникает за счет $\sigma$-связей электронов исследуемого атома с лигандами. В рентгеновских спектрах азота в нитратах выявлена структура резонансных уровней (плазмонов), возникающих в низкоэнергетичной части зоны проводимости и участие их в косвенном обменном взаимодействии.

В рентгеновских спектрах наряду с косвенным обменным взаимодействием проявляется широкий круг неупругих взаимодействий, связанных друг с другом: неупругие столкновения выбитых фотоэлектронов, возбуждение плазмонных сателлитов в рентгеноэлектронных спектрах [19] и в первичных эмиссионных спектрах [23], резонансы в спектрах поглощения, локализованные в зоне проводимости и близкие к резонансам формы [26] и другие. Для этих процессов очень существенна роль рентгеновской вакансии. Спектры дают информацию, отвечающую среднему по состояниям системы, включающей исследуемый атом и атомы ближайших соседей, а не среднему по времени. Совокупность этих процессов позволяет изучать связь между состояниями электронной, спиновой и атомной подсистем с данными о магнитной структуре.

\section{Конфликт интересов}

Авторы заявляют, что у нас нет конфликта интересов.

\section{Список литературы}

[1] Л.М. Волкова. Журн. структур. химии. 50, 7, S55 (2009).

[2] A.N. Vasilev, M.M. Markina, E.A. Popova. Low Temper. Phys. 31, 3-4, 203 (2005).

[3] А.С. Боровик-Романов, Н.М. Крейнес, А.А. Панков, М.А. Талалаев. ЖЭТФ 66, 2, 782 (1974).

[4] O.S. Volcova, V.V. Mazurenko, I.V. Solovyev, E.B. Deeva, I.V. Morozov, J.-Y. Lin, C.K. Wen, J.M. Chen, M. Abdel-Hafie, A.N. Vasiliev. Phys. Rev. B 90, 134407 (2014).

[5] I. Tudosa, C. Stamm, A.B. Kashuba, F. King, H.C. Siegmann, J. Stöhr, G. Ju, B. Lu, D. Weller. Nature 428 (6985), 831 (2004).

[6] А.С. Боровик-Романов, М.П. Орлова. ЖЭТФ 31, 4 (10), 579 (1956)

[7] Е.А. Туров. Физические свойства магнитоупорядоченных кристаллов. Изд. АН СССР, М. (1963). 223 с.

[8] А.И. Дмитриев, О.В. Коплак, А. Namai, Н. Tokoro, S. Ohkoshi, Р.Б. Моргунов. ФТТ 55, 11, 2140 (2013).

[9] И.Е. Дзялошинский. ЖЭТФ 32, 1547 (1957).

[10] У.Л. Брэгг, Г.Ф. Кларингбулл. Кристаллическая структура минералов. Мир. М. (1967). 391 с.

[11] Ю.Н. Журавлев, А.С. Поплавной. Журн. структур. химии. 44, 3, 553 (2003).

[12] Р.Л. Баринский, И.В. Кошелева, И.М. Куликова. Журн. структур. химии 30, 6, 122 (1989). 
[13] Р. Уайт. Квантовая теория магнетизма. Мир М. (1985). $303 \mathrm{c.}$

[14] Г.В. Базуев, А.В. Королев, Б.Г. Головкин. ФТТ 58, 7, 1289 (2016).

[15] Р.Л. Баринский, И.М. Куликова, Е.П.Шевченко. Изв. АН СССР. Сер. физ. 46, 4, 724 (1982).

[16] Л.Н. Мазалов, А.Д. Федоренко, Н.А. Крючкова, Ю.П. Диков, Ю.Ф. Полиенко, Н.А Григорьев. Журн. структур. химии 55, 6, 1100 (2014).

[17] И.М. Куликова, О.А. Набелкин, Ю.Г. Лаврентьев, В.А. Иванов. Завод. лаб. Диагностика материалов 85, 11, 5 (2019).

[18] Е.В. Стукова. Изв. РАН. Сер. физ. 77, 8, 1122 (2013).

[19] В.И. Нефедов, Рентгеноэлектронная спектроскопия химических соединений. изд. Химия, М. (1984). 255 с.

[20] V.I. Nefedov, Ju.A. Buslaev, N.P. Sergushin, K. Bayer, Ju.V. Kokunov, V.V. Kovalev. J. Electron Spectrosc. 6, 221 (1975).

[21] Ю.Н. Журавлев, А.С. Поплавной. Журн. структур. химии 42, 6, 1056 (2001).

[22] И.А. Федоров, Ю.Н. Журавлев, Д.В. Корабельников. Изв. вузов. Физика. 49, 10, 61 (2006).

[23] И.М. Куликова, О.А. Набелкин, В.А. Иванов. Журн. структур. химии. 61, 11, 1795 (2020).

[24] В.И. Нефедов, М.А. Порай-Кошиц. Журн. структур. химии 13, 5, 864 (1972).

[25] М.Б. Миклин. Вестн. Кемеровского гос. ун-та. 3, 3 (59), 234 (2014).

[26] С.В. Некипелов, А.С. Виноградов, В.Н. Сивков. Изв. Коми научного центра УрО РАН 2, 6, 12 (2011).

[27] V.B. Gildenburg, V.A. Kostin, I.A. Pavlichenko. Phys. Plasmas 18, 092101 (2011).

[28] V.B. Gildenburg, I.A. Pavlichenko, A.I. Simatov. Phys. Plasmas 21, 012301 (2014).

Редактор Ю.Э. Китаев 